\title{
Sitaxentan-related acute liver failure in a patient with pulmonary arterial hypertension
}

\section{To the Editors:}

Liver toxicity is a well recognised side-effect of endothelin receptor antagonists (ERAs) (bosentan, sitaxentan and ambrisentan) and long-term monthly monitoring of liver function tests (LFTs) is mandatory. Sitaxentan is thought to be less commonly associated with liver toxicity compared with the older agent bosentan and has been used as an alternative in patients who develop liver dysfunction on bosentan [1-3]. However, several recently published cases of sitaxentan-related liver injury have raised concerns that the potential of sitaxentan to cause serious liver toxicity has been underestimated. To date, there are six such cases reported in the medical literature, including two deaths (one patient was on a higher dose of $300 \mathrm{mg}$ twice daily in the pre-marketing stage and the other patient died from pneumonia 2 months after drug withdrawal) and one patient requiring liver transplantation (patient was also on another potentially hepatotoxic drug) [4-6]. We would like to report a case of a young patient with idiopathic pulmonary arterial hypertension (IPAH) who developed acute liver failure while taking sitaxentan. Her LFTs had always been and were normal, last tested 1 month before the development of acute liver failure, which was within the timescale of the LFT monitoring procedure currently recommended.

This 19-yr-old patient diagnosed with severe IPAH was treated with sildenafil $50 \mathrm{mg}$ three times daily with an initial response. She had no other comorbid conditions, in particular had no preexisting liver disease. Her exertional symptoms progressed a few months later and sitaxentan $100 \mathrm{mg}$ daily was added. 3 months later, she was noted to have developed jaundice overnight. Sitaxentan was discontinued immediately. On admission to the Golden Jubilee National Hospital (Glasgow, UK) 2 days later, she was markedly jaundiced with grossly deranged liver function and mild coagulopathy. Bilirubin was elevated at $177 \mathrm{mg} \cdot \mathrm{dL}^{-1}$, alkaline phosphatase $188 \mathrm{IU} \cdot \mathrm{L}^{-1}, \gamma$-glutamyltransferase $147 \mathrm{IU} \cdot \mathrm{L}^{-1}$, aspartate transaminase $1,300 \mathrm{IU} \cdot \mathrm{L}^{-1}$, alanine transaminase $1,250 \mathrm{IU} \cdot \mathrm{L}^{-1}$ and prothrombin time $16 \mathrm{~s}$. There were no immune features such as fever, skin rash, eosinophilia or autoantibodies. Viral hepatitis (hepatitis A, B, C and E) screen was negative. Serum caeruloplasmin and copper levels were normal. Abdominal ultrasound showed normal liver, normal biliary tract, patent hepatic and portal veins. In addition to sildenafil, other medication included omeprazole, domperidone and amoxycillin, which were prescribed by her general practitioner for an ear infection a few days prior to the onset of jaundice. She had previously been exposed to amoxycillin with no adverse reaction. She was not on oral anti-coagulant therapy due to a history of syncope and concerns about the risk of traumatic haemorrhage. LFTs 1 month prior to the onset of jaundice were normal (bilirubin $10 \mathrm{mg} \cdot \mathrm{dL}^{-1}$, alkaline phosphatase $68 \mathrm{IU} \cdot \mathrm{L}^{-1}$ and alanine transaminase $\left.26 \mathrm{IU} \cdot \mathrm{L}^{-1}\right)$.

Her LFTs failed to improve after withdrawal of sitaxentan. They remained relatively static for 4 days, but then gradually worsened over the following few days. On day 8, her prothrombin time rose to $24.5 \mathrm{~s}$ and bilirubin to $424 \mathrm{mg} \cdot \mathrm{dL}^{-1}$, indicative of impending fulminant hepatic failure. She was transferred to the regional lung and liver transplant centre, Freeman Hospital (Newcastle upon Tyne, UK), for urgent liver transplantation. Prednisolone $50 \mathrm{mg}$ daily was initiated at this stage. Although a donor liver became available, she developed refractory cerebral oedema and died on day 13. Post mortem examination showed that her liver had undergone acute extensive necrosis, with a vigorous stem cell regenerative response as a result of a drug reaction (fig. 1). There was no evidence of pre-existing fibrosis or cirrhosis (fig. 2). Staining for copper and copper-associated protein with orcein and rhodanine showed no evidence of copper deposition, excluding the possibility of Wilson's disease.

Sitaxentan is implicated as a possible cause of acute liver failure in this case, but its definitive role cannot be determined due to co-administration of omeprazole, domperdone and amoxycillin prior to the onset of jaundice. The possibility that omeprazole and/or amoxycillin had caused or contributed to liver injury cannot be excluded as they had been previously reported to cause acute liver injury $[7,8]$. However, proton pump inhibitors were not identified as hepatotoxic in a large ongoing prospective multicentre epidemiological study in the USA [9]. Another community-based case-control study in the UK showed that amoxycillin without clavulanic acid was nonhepatotoxic after adjusting for concomitant hepatotoxic drug exposure [10]. Conversely, sitaxentan is known to cause hepatic dysfunction, although it is usually asymptomatic and self-limiting [11]. Another risk factor of hepatotoxicity is drug

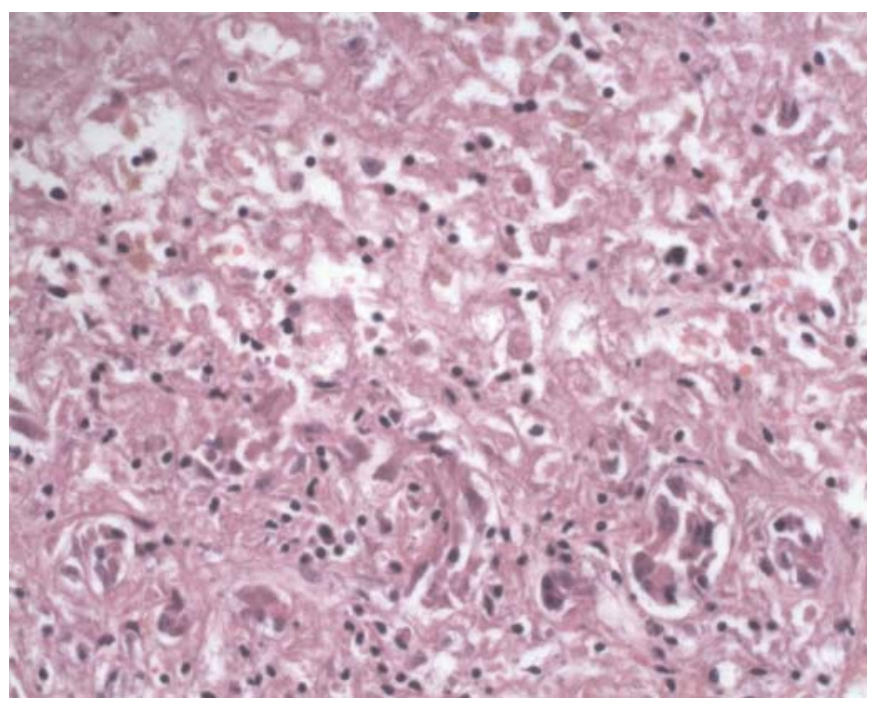

FIGURE 1. Post mortem liver histology showing extensive diffuse liver necrosis with a vigorous stem cell regenerative response (bottom right). Haematoxylin and eosin staining. $\times 400$ magnification 


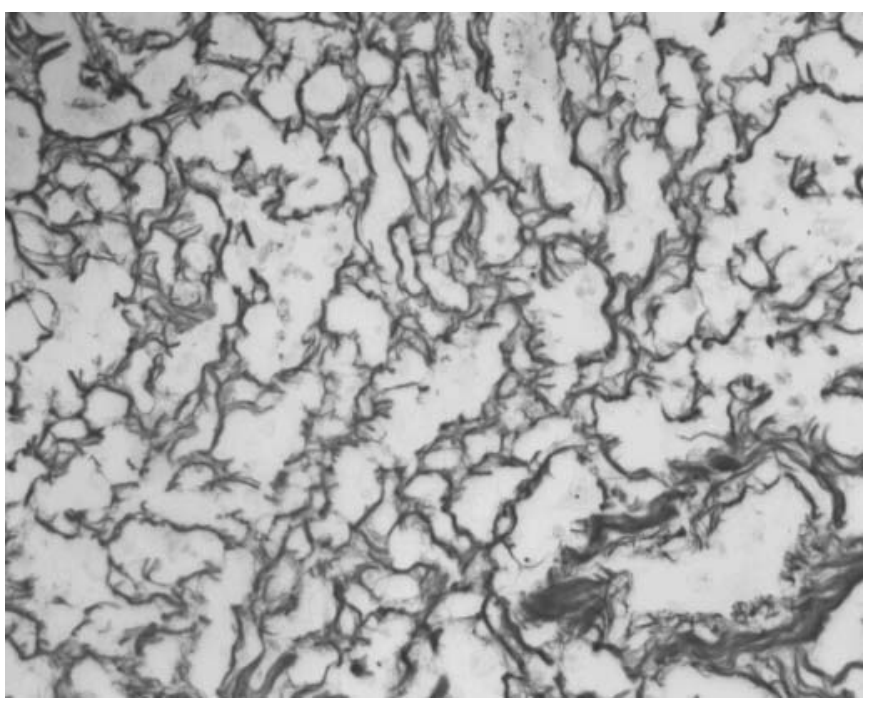

FIGURE 2. Reticulin staining showing that the sinusoidal architecture is preserved confirming the normal nature of the pre-morbid liver. $\times 400$ magnification.

interaction leading to altered pharmacokinetics. Sitaxentan is an inhibitor of cytochrome P450 isoenzyme CYP2C9 and, to a lesser degree, CYP2C19, CYP3A4/5 and CYP2C8 [12]. Although plasma concentrations of drugs principally metabolised by CYP2C9 may be increased during sitaxentan co-administration, concurrent use of drugs metabolised by CYP2C19 or CYP3A4/5 is not expected to result in clinically significant drug interactions, which would be the case for omeprazole (a substrate of CYP2C19), domperidone (a substrate of CYP3A4) and sildenafil (a substrate of CYP3A4). Amoxycillin is not directly metabolised by cytochrome P450 enzymes and, therefore, is not expected to interact with sitaxentan.

Idiosyncratic acute liver failure due to any drug may only be observed in the post-marketing stage due to its rare occurrence [13]. The precise mechanisms remain poorly understood. Susceptibility to drug toxicity varies greatly among individuals due to the highly complex interplay between genetic, immunological and environmental factors [14]. Genetic polymorphism of cytokines, hepatic enzymes and transcription factors, human leukocyte antigen genotype and individual variation in innate and adaptive immune responses have been shown to play a role. Environmental factors such as preexisting liver disease, alcohol use, concomitant infections or inflammation and drug interactions may act as a trigger for hepatotoxicity in the presence of other intrinsic risk factors. In this case, the only established risk factor, apart from concurrent drug use, is female sex [15]. Subclinical hepatic congestion due to pulmonary arterial hypertension and right heart dysfunction could theoretically alter the "environment" and predispose the liver to drug toxicity. However, post mortem examination confirmed that the pre-morbid liver was normal and there was no evidence of hepatic congestion.

Although the definitive cause of acute liver failure in this case cannot be proven, it reinforces the ongoing concern of hepatotoxicity associated with the use of ERAs. Published post-marketing surveillance data on 4,994 patients treated with bosentan in Europe over a period of 30 months showed that the incidence of raised aminotransferases was 7.6\% [16]. Reassuringly, only 10 cases of serious liver injury were reported with no fatality. For the newer agents sitaxentan and ambrisentan, the collection of post-marketing safety data is still ongoing. As more patients with pulmonary arterial hypertension are diagnosed and treated, the use of ERAs is expected to rise. It is vital that clinicians are vigilant against potential hepatotoxicity and exercise more caution when prescribing other medication in patients already on ERAs. Patients should be given explicit advice on the importance of LFT monitoring and the nonspecific features of hepatotoxicity so that they may present earlier before jaundice is manifest.

\section{W-T.N. Lee*, N. Kirkham", M.K. Johnson*, J.L. Lordan", A.J. Fisher and A.J. Peacock*}

*Scottish Pulmonary Vascular Unit, Golden Jubilee National Hospital, Glasgow, and "Dept of Cellular Pathology, Royal Victoria Infirmary, and "Dept of Cardiopulmonary Transplantation, Freeman Hospital, Newcastle upon Tyne, UK.

Correspondence: A.J. Peacock, Scottish Pulmonary Vascular Unit, Level 1, Golden Jubilee National Hospital, Beardmore Street, Clydebank, Glasgow G81 4HX, UK. E-mail: apeacock@ udcf.gla.ac.uk

Statement of Interest: Statements of interest for all authors of the present study can be found at www.erj.ersjournals.com/ site/misc/statements.xhtml

Acknowledgement: We would like to thank A. Daly (Institute of Cellular Medicine, Newcastle University, Newcastle upon Tyne, UK) for her expert advice.

\section{REFERENCES}

1 Barst RJ, Langleben D, Badesch D, et al. Treatment of pulmonary arterial hypertension with the selective endothelin-A receptor antagonist sitaxsentan. J Am Coll Cardiol 2006; 47: 2049-2056.

2 Benza RL, Mehta S, Keogh A, et al. Sitaxsentan treatment for patients with pulmonary arterial hypertension discontinuing bosentan. J Heart Lung Transplant 2007; 26: 63-69.

3 Dupuis J, Hoeper MM. Endothelin receptor antagonists in pulmonary arterial hypertension. Eur Respir J 2008; 31: 407-415.

4 Barst RJ, Rich S, Widlitz A, et al. Clinical efficacy of sitaxsentan, an endothelin-A receptor antagonist, in patients with pulmonary arterial hypertension: open-label pilot study. Chest 2002; 121: 1860-1868.

5 Hoeper MM, Olsson KM, Schneider A, et al. Severe hepatitis associated with sitaxentan and response to glucocorticoid therapy. Eur Respir J 2009; 33: 1518-1519.

6 Lavelle A, Sugrue R, Lawler G, et al. Sitaxentan-induced hepatic failure in two patients with pulmonary arterial hypertension. Eur Respir J 2009; 34: 770-771.

7 Garcia Rodriguez LA, Wallander MA, Stricker BH. The risk of acute liver injury associated with cimetidine and other acidsuppressing anti-ulcer drugs. Br J Clin Pharmacol 1997; 43: 183-188.

8 Sabate M, Ibanez L, Perez E, et al. Risk of acute liver injury associated with the use of drugs: a multicentre population survey. Aliment Pharmacol Ther 2007; 25: 1401-1409.

9 Chalasani N, Fontana RJ, Bonkovsky HL, et al. Causes, clinical features, and outcomes from a prospective study of drug-induced liver injury in the United States. Gastroenterology 2008; 135: 1924-1934. 
10 de Abajo FJ, Montero D, Madurga M, et al. Acute and clinically relevant drug-induced liver injury: a population based casecontrol study. Br J Clin Pharmacol 2004; 58: 71-80.

11 Hoeper MM. Liver toxicity: the Achilles' heel of endothelin receptor antagonist therapy? Eur Respir J 2009; 34: 529-530.

12 The electronic Medicines Compendium (eMC). www.medicines. org.uk. Date last updated: August 3, 2010. Date last accessed: August 3, 2010.

13 Verma S, Kaplowitz N. Diagnosis, management and prevention of drug-induced liver injury. Gut 2009; 58: 1555-1564.
14 Russmann S, Kullak-Ublick GA, Grattagliano I. Current concepts of mechanisms in drug-induced hepatotoxicity. Curr Med Chem 2009; 16: 3041-3053.

15 Lee WM, Squires RH Jr, Nyberg SL, et al. Acute liver failure: summary of a workshop. Hepatology 2008; 47: 1401-1415.

16 Humbert M, Segal ES, Kiely DG, et al. Results of European postmarketing surveillance of bosentan in pulmonary hypertension. Eur Respir J 2007; 30: 338-344.

DOI: $10.1183 / 09031936.00091610$ 